\title{
Numerical Solution of Stiff and Singularly Perturbed Boundary Value Problems With a Segmented-Adaptive Formulation of the Tau Method
}

\author{
By P. Onumanyi and E. L. Ortiz
}

To Professor Y. L. Luke, in memoriam

\begin{abstract}
This paper concerns the application of Ortiz' recursive formulation of the Tau method to the construction of piecewise polynomial approximations to the solution of linear and nonlinear boundary value problems for ordinary differential equations. A practical error estimation technique, related to the concept of correction in Zadunaisky's sense, is considered and used in the design of an adaptive approach to the Tau method. It proves efficient in the numerical treatment of problems with rapid functional variations, stiff and singularly perturbed problems. A technique of increased accuracy at matching points of segmented Tau approximants is also discussed and successfully applied to several problems. Numerical examples show that, for a given degree of approximation, our segmented Tau approximant gives an accuracy comparable to that of the best segmented approximation of the exact solution by means of algebraic polynomials.
\end{abstract}

1. Introduction. We discuss the use of Ortiz' recursive formulation of the Tau method [23]-[25] in the numerical solution of boundary value problems for linear and nonlinear differential equations defined over an interval $a \leqslant x \leqslant b$. We consider global approximations over $[a, b]$, with a single polynomial expression, and segmented forms based on a step-by-step formulation of the Tau method considered by Ortiz in [26].

The Tau approximate solution of a differential problem defined by a differential operator $D$ is represented in terms of the elements of a sequence $Q$ of canonical polynomials. Such a sequence is uniquely determined by $D$, it is independent of the specific boundary conditions of the problem, and of the particular interval $[a, b]$ in which the solution is required. These properties make possible the use of segmentation within the framework and with the software [32] designed for the recursive formulation of the Tau method. The concept of correction, in Zadunaisky's sense [37] (see also Stetter [36]), is discussed in the context of the Tau method and related to a practical error estimation technique. This technique, based on Tau estimators introduced here, is systematically applied to all examples, linear or nonlinear. It is

Received February 11, 1982; revised August 30, 1983.

1980 Mathematics Subject Classification. Primary 65L05, 65L10, 65N35.

Key words and phrases. Initial value problems, boundary value problems, systems of ordinary differential equations, stiff problems, singularly perturbed problems, step-by-step approximation, adaptive methods, Tau method. 
also used in the control of the segmentation process: an adaptive form of the Tau method, based on this, is considered.

A technique of increased accuracy at matching points of segmented Tau approximations, introduced by Ortiz in [27] for initial value problems, is successfully used in linear and nonlinear boundary value problems. Numerical comparisons with other standard methods and, in particular, with an accurate technique of collocation with splines followed by a finite difference correction (see [18]) is definitely favorable to our approach. We find that, for a given degree of approximation, our segmented Tau approximants are close to the best segmented approximations by algebraic polynomials. This optimal result has been reported for the global case by Freilich and Ortiz in a recent paper [6].

Convergence results and error bounds for Tau approximations of the solution of differential equations are discussed by Luke [13], Lanczos [10]-[12], and Ortiz and Pham [30]-[31]. Systems of differential equations have been discussed by Freilich and Ortiz [6] and Crisci and Russo [3]. The effect of approximating the coefficients of a differential equation on the accuracy of the Tau approximant is discussed by Namasivayam and Ortiz in [17]. An interesting feature of the Tau method is the fact that no trial solutions, approximate quadratures or large matrix inversions are required. A summary of the results of this paper was presented to the Dundee Conference of Numerical Analysis, June 1981.

2. Recursive Formulation of the Tau Method: Some Basic Definitions and Notation. Let $\mathbf{P}_{j}$ be the class of polynomials of degree less than or equal to $j$. We shall consider the equation defined by the differential operator $D$ :

$$
D y(x):=p_{\nu}(x) y^{(\nu)}(x)+\cdots+p_{1}(x) y^{(1)}(x)+p_{0}(x) y^{(0)}(x)=f(x),
$$

where either $p_{i}(x) \in \mathbf{P}_{\alpha_{i}}, i=0(1) \nu$, and $f(x) \in \mathbf{P}_{F}$, or they are close polynomial approximations of given functions. The symbol $y^{(i)}(x)$ stands for the $i$ th derivative of $y(x)$, and $y^{(0)}(x) \equiv y(x)$. The solution $y(x)$ of (1) satisfies boundary conditions of the general form

$$
\sum_{i=0}^{\nu-1}\left[a_{r i} y^{(i)}(a)+b_{r i} y^{(i)}(b)\right]=A_{r}, \quad r=1(1) \nu,
$$

where some of the coefficients $a_{r i}, b_{r i}$ may be equal to zero. We shall follow Ortiz' algebraic theory of the Tau method, of which we will give here some basic definitions and results. Further details and proofs can be found in [23]-[25], and [14].

A useful number associated with any differential operator $D$ of the class $\mathscr{D}$, characterized by (1), is its height $h$,

$$
h:=\max _{0 \leqslant i \leqslant \nu}\left(\alpha_{i}-i\right) .
$$

Each differential operator $D \in \mathscr{D}$. is uniquely associated with a sequence $Q$ of canonical polynomials $Q_{n}(x)$ defined for all indices $n \in \mathbf{N}-S$. The set $\mathbf{N}:=\{0,1,2,3, \ldots\} ; S$ is finite, usually very small, and the number $s$ of its elements is bounded by $\nu+h$. The polynomials $Q_{n}(x)$ satisfy the functional equation

$$
D Q_{n}(x)=x^{n}+r_{n}(x), \quad n \in \mathbf{N}-S,
$$


where $r_{n}(x) \in \mathbf{R}_{S}=\operatorname{span}_{i \in S}\left\{x^{i}\right\}$ is called the residual of $Q_{n}(x)$. If all powers of $x$ can be obtained as images of polynomials under the differential operator $D$, then $S$ will be empty. If $D$ is applied to the monomial $x^{m}$, we obtain the generating polynomials

$$
D x^{m}=\sum_{i=0}^{n} a_{i} x^{i}, \quad m \in \mathbf{N},
$$

from which a recursive relation, involving at most $\nu+h$ (assuming $p_{0}(x) \equiv 0$ ) canonical polynomials, can immediately be deduced if $a_{n} \neq 0$ (see Ortiz' Theorem 3.3 in [24]). Otherwise, (4) is used to find the minimal set $S$ of indices of undefined canonical polynomials and $\operatorname{Ker}(D)$, the algebraic kernel of $D$, which contains all the exact polynomial solutions of (1). The recursive relation for the $Q_{n}(x)$ 's is self starting and the polynomials themselves depend neither on the supplementary conditions ((2) in our case) imposed on $y(x)$, nor on the interval in which the solution is required. These properties will be used in the design of a segmented formulation of the Tau method of [26] for boundary value problems.

Let $\mathbf{v}=\left\{v_{i}(x)\right\}=V \mathbf{x}$ be a polynomial basis defined by a lower triangular matrix $V=\left(\left(v_{i j}\right)\right), i, j \in \mathbf{N}$, acting on $\mathbf{x}=\left(1, x, x^{2}, \ldots\right)^{\prime}$. Clearly $\hat{Q}=\left\{\hat{Q}_{n}(x)\right\}, n \in \mathbf{N}-$ $S$, is such that $D \hat{Q}_{n}(x)=v_{n}(x)+\hat{r}_{n}(x), \hat{r}_{n}(x) \in \mathbf{R}_{S}$, if

$$
\hat{Q}_{n}(x)=\sum_{j=0}^{n} v_{n j} Q_{j}(x), \quad \text { with } j \notin S .
$$

Definition 1. A polynomial $g(x)$ is called a Tau approximant of order $n$ of $y(x)$ if it satisfies exactly the differential equation (1) with a polynomial perturbation term $H_{n}(x) \in \mathbf{P}_{n}$, and if it satisfies exactly boundary conditions (2).

Clearly $g(x) \in \mathbf{P}_{n-h}$. Let

$$
H_{n}(x)=\sum_{i=0}^{m} \tau_{i}^{(n)} v_{n-i}(x) \in \mathbf{P}_{n}
$$

be the perturbation term, expressed in the basis $\mathbf{v}$, and let $\tau_{i}^{(n)}, i=0(1) m$, be free parameters. Let us assume further that

$$
f(x)=\sum_{i=0}^{F} f_{i} v_{i}(x),
$$

and that $n$ is chosen sufficiently large for $F \leqslant n$. Then,

$$
y_{n}(x):=\sum_{i=0}^{m} \tau_{i}^{(n)} \hat{Q}_{n-i}(x)+\sum_{i=0}^{F} f_{i} \hat{Q}_{i}(x), \quad \text { with } i \notin S,
$$

satisfies the equation $D y_{n}(x)=H_{n}(x)+f(x)$ if $s$ parameters $\tau_{i}^{(n)}$ are chosen in such a way that the residuals of $D y_{n}(x)$ match the components of $H_{n}(x)+f(x)$ belonging to $\mathbf{R}_{S}$. If $\nu$ further parameters $\tau_{i}^{(n)}$ are fixed for $y_{n}(x)$ to satisfy exactly the boundary conditions (2), then $y_{n}(x)$ is a Tau approximant of order $n$ of $y(x)$. If there exist $t$ exact polynomial solutions of (1), then $m=s+\nu-t-1$. 
Finally, if (1) is given in an integrated form, through an integral operator $I$, a sequence $\bar{Q}=\left\{\bar{Q}_{n}(x)\right\}, n \in \mathbf{N}-S_{I}$, such that $I \bar{Q}_{n}(x)=x^{n}+\bar{r}_{n}(x), \bar{r}_{n}(x) \in \mathbf{R}_{S_{I}}$ can be constructed immediately in terms of the sequence $Q$ associated with $D$ :

$$
\bar{Q}_{n}(x)=\frac{n !}{(n-\nu) !} Q_{n-\nu}(x), \quad \text { with } S_{I}=\{n \in \mathbf{N}: n<\nu, n-\nu \in S\} .
$$

A variety of choices of basis $\mathbf{v}$ is possible in the Tau method. If $\mathbf{v}$ is $\left\{x^{n}\right\}, n \in \mathbf{N}$, the Tau method realizes the power series expansion method, where a high accuracy is to be expected near the point of expansion. Lanczos [11]-[12] suggested the choice of Chebyshev and Legendre polynomials to obtain a better distribution of errors in the equation over the interval in which the approximate solution is required. The error in the equation, namely $H_{n}(x)$, is related to the error of approximation

$$
e_{n}(x)=y_{n}(x)-y(x)
$$

through the inverse operator of $D: D^{-1} H_{n}(x)=e_{n}(x)$, on account of the linearity of $D$. The choice of $H_{n}(x)$ close to a best uniform approximation of the function identically equal to zero in $[a, b]$ by means of algebraic polynomials is an attempt to take advantage of the relation between $H_{n}(x)$ and $e_{n}(x)$. However, that relation requires a careful analysis if quantitative results are required. The behavior of $e_{n}(x)$ relative to the perturbation term $H_{n}(x)$ is discussed by Namasivayam and Ortiz [16].

Remark 1. If the solution $y(x)$ of (1)-(2) is a polynomial of degree $k$, any Tau approximate solution of degree $\geqslant k$ will detect it exactly. In this sense we say that the Tau method is exact of degree $k$.

3. Correction of Tau Approximants. We now consider the correction technique of Zadunaisky [37] and Stetter [36] in the context of the Tau method.

THEOREM 1. Correction of a Tau approximant leads to a Tau approximant of a higher order.

Proof. Let us consider the error function $e_{n}(x)$. From (1) it follows that $e_{n}(x) \in$ $C^{(\nu)}[a, b]$ and that it satisfies the differential equation $D e_{n}(x)=H_{n}(x)$, where all $\tau$-parameters in $H_{n}(x)$ are fixed. It also satisfies boundary conditions (2) with $A_{r}=0$, for $r=1(1) \nu$.

Let $\left[e_{n}(x)\right]_{m}$ be an $m$ th order $(m>n)$ Tau approximant of the error function. Then the polynomial $\left[e_{n}(x)\right]_{m}$ satisfies the differential equation

$$
D\left[e_{n}(x)\right]_{m}=H_{n}(x)+H_{m}(x)=f(x)+H_{n}(x)-\left[f(x)-H_{m}(x)\right],
$$

with homogeneous boundary conditions (2). Let $y_{m}(x)$ be the $m$ th order Tau approximant of $y(x)$. Then, $e_{n}(x)-e_{m}(x)=y_{n}(x)-y_{m}(x)$. From the uniqueness of the Tau approximant for a given order $m$ (see Ortiz [24]) and (9), it follows that $\left[e_{n}(x)\right]_{m}=e_{n}(x)-e_{m}(x)$. Thus, $y_{n}(x)$ plus the correction term $\left[e_{n}(x)\right]_{m}$ equals $y_{m}(x)$.

Definition 2. $\left[e_{n}(x)\right]_{m}$ defined by (9) is called the Tau estimator of order $n, m$. Examples of Tau approximants corrected with the Tau estimator, for $m \geqslant n+1$, are given in the last section of this paper. 


\section{Implicit Matching of Tau Approximants. Let}

$$
\Pi=\left\{x_{0}=a<x_{1}<x_{2}<\cdots<x_{p-1}<x_{p}=b\right\}
$$

be a partition of the interval $[a, b]$ into subintervals $\left[x_{j-1}, x_{j}\right], j=1(1) p$,

Definition 3. The vector

$$
\mathbf{y}_{n}(x)=\left[y_{n 1}(x), y_{n 2}(x), \ldots, y_{n p}(x)\right],
$$

with $y_{n j}(x)$ defined for $x_{j-1} \leqslant x \leqslant x_{j}, j=1(1) p$, is a piecewise Tau approximant of order $n$ of the solution $y(x)$ of the boundary value problem (1)-(2) if each of the $y_{n j}(x), j=1(1) p$, satisfies (1) with a polynomial perturbation term $H_{n}^{j}(x)$ defined for $x_{j-1} \leqslant x \leqslant x_{j}$, and

$$
\begin{gathered}
\sum_{i=0}^{\nu-1}\left[a_{r i} y_{n 1}^{(i)}(a)+b_{r i} y_{n p}^{(i)}(b)\right]=A_{r} \quad \text { for } r=1(1) \nu, \\
y_{n j-1}^{(i)}\left(x_{j-1}\right)=y_{n j}^{(i)}\left(x_{j-1}\right) \quad \text { for } j=2(1) p, i=0(1) \nu-1 .
\end{gathered}
$$

Remark 2. Condition (11-a) imposes on $\mathbf{y}_{n}(x)$ the $\nu$ boundary conditions given by (2), while (11-b) is a continuity requirement for $y_{n j}(x)$ and its $\nu-1$ derivatives at the $p-1$ interior partition points of $\Pi$. If $S \neq \varnothing$, matching coefficients of terms belonging to $\mathbf{R}_{S}$ in the right-hand side of the differential equation with the residuals of $D y_{n j}(x)$ provides us with $s$ conditions in each of the $p$ subintervals defined by the partition. Thus, a total of $p(s+\nu)$ conditions.

THEOREM 2. The construction of an nth order piecewise Tau approximation $\mathbf{y}_{n}(x)$ of the solution $y(x)$ of boundary value problem (1)-(2) depends on only one matrix $V$ and one canonical sequence $Q$.

Proof. Let us consider the family of mappings $\mathscr{S}_{j}, j=1(1) p$ (see Ortiz [26]) acting on $y_{n}(x)$ of (7) and such that

$$
\mathscr{S}_{j} y_{n}(x)=y_{n j}(x), \quad x_{j-1} \leqslant x \leqslant x_{j},
$$

and let us call $\tau_{i j}^{(n)}$ the free parameters corresponding to $y_{n j}(x)$. Without loss of generality, let us assume that $a=0$, and let $E_{j}=1 /\left(x_{j}-x_{j-1}\right), j=1(1) p$. The mappings $\mathscr{S}_{j}$ admit a trivial realization: replacing $v_{n j}$ by $v_{n i}^{j}=v_{n i}\left(E_{j}\right)$, we find that $\left(\left(v_{n i}^{j}\right)\right)$ defines the basis $\mathbf{v}^{j}$ corresponding to the interval $x_{j-1} \leqslant x \leqslant x_{j}$, for $j=1(1) p$. Taking into account (7) the result is proved.

We now discuss the computational implications of Theorem 2. Let $\tau_{j}^{(n)}=\left(\tau_{i j}^{(n)}\right)^{\prime}$, $i=0(1) s+\nu-1$, be a vector, the elements of which are the Tau parameters of the component $y_{n j}(x)$ of $y_{n}(x)$, for $j=1(1) p$, and let $\tau^{(n)}=\left(\tau_{1}^{(n) \prime}, \ldots, \tau_{p}^{(n) \prime}\right)^{\prime}$ be a vector with $p(s+\nu)$ components. Let $a=x_{j-1}, b=x_{j}$. Then (7) gives us $y_{n j}(x)$ for $j=1(1) p$. The piecewise polynomial Tau approximant $\mathbf{y}_{n}(x)$ of $y(x)$ will be determined if $\tau^{(n)}$ is found.

From Remark 2 it follows that $\tau^{(n)}$ is implicitly defined by a system of linear algebraic equations of the form

$$
Z_{n} \tau^{(n)}=\mathbf{W}_{n} .
$$

Let us split $y_{n j}(x)$ (see (7), with $a=x_{j-1}, b=x_{j}$ ) into two parts: one dependent on the Tau parameters, and then related to $Z_{n}$, and the other independent of them, thus 
related to $\mathbf{W}_{n}$. We shall write $\phi_{n j}(x) \tau_{j}^{(n)}$ for the vector with $\nu$ rows, containing as elements that first part of $y_{n j}(x)$ and its $\nu-1$ derivatives. Let $\xi_{n j}(x)$ be a vector containing in each of its rows the second part of $y_{n j}(x)$ and its $\nu-1$ derivatives. The residual $D y_{n j}(x)$ of $y_{n j}(x)$ belongs to $\mathbf{R}_{S}$, which is a subspace generated by $s$ linearly independent basis elements. We will split the residual of $y_{n j}(x)$ in the same way as before, placing the $s$ components in $\mathbf{R}_{S}$ of the first part, dependent on the Tau terms, in successive rows of a vector $\rho_{n j} \tau_{j}^{(n)}$ and the rest of it in the successive rows of a vector $\eta_{n j}$.

With the help of matrices $\phi_{n j}$ and $\rho_{n j}$, of orders $\nu \times(s+\nu)$ and $s \times(s+\nu)$, respectively, we can discuss the structure of matrix $Z_{n}$ of (12). $Z_{n}$ is made up of blocks $\alpha_{j}^{(n)}$, each one of them related to one component of $\mathbf{y}_{n}$, for $j=1(1) p$. These blocks have the form

$$
\alpha_{j}^{(n)}=\frac{\phi_{n j}\left(x_{j-1}\right)}{\rho_{n j}} \text { for } j=2(1) p-1
$$

in $\alpha_{p}^{(n)}$ the third subblock is missing, and $\alpha_{1}^{(n)}$ has the same second and third subblocks, but a different first subblock $\gamma_{n 1}$. Let

$\mathscr{A}=\left(\left(a_{r i}\right)\right), \mathscr{B}=\left(\left(b_{r i}\right)\right), \quad r=1(1) \nu, i=0(1) \nu-1$, and $\mathbf{A}=\left(A_{1}, \ldots, A_{\nu}\right)^{\prime}$,

where $a_{r i}, b_{r i}$, and $A_{i}$ are the coefficients of the boundary conditions (11-a); then $\gamma_{n 1}$ is such that

$$
\gamma_{n 1} \tau^{(n)}=\mathscr{A} \phi_{n 1}(a) \tau_{1}^{(n)}+\mathscr{B} \phi_{n p}(b) \tau_{p}^{(n)}
$$

Matrix $Z_{n}$ is constructed by linking the blocks $\alpha_{j}^{(n)}$ in such a way that the third subblock of $\alpha_{j}^{(n)}$ faces the first subblock of $\alpha_{j+1}^{(n)}$ for $j=1(1) p-1$. The first $\nu$ components of $\mathbf{W}_{n}$ have the following form:

$$
\mathbf{A}-\mathscr{A} \xi_{n 1}(a)-\mathscr{B} \xi_{n p}(b)
$$

with this choice the boundary conditions (11-a) are incorporated into (12). The remaining elements of $\mathbf{W}_{n}$ follow if $\xi_{n j}$ and $\eta_{n j}$ are assembled following the same rules as for the corresponding Tau-dependent elements in $Z_{n}$. The rows of $Z_{n}$ with $\phi$ 's realize the continuity conditions (11-b), while the rows with $\rho$ 's make sure that the sum of residuals of each component $y_{n j}$ match the terms in $\mathbf{R}_{S}$ on the right-hand side of the Tau problem for each $x_{j-1} \leqslant x \leqslant x_{j}, j=1(1) p$. We have assumed that $t=0$, if this is not the case, only $s+\nu-t$ Tau parameters are required, as $t$ free parameters are provided by the exact polynomial solutions of $D y(x)=0$.

Remark 3. The following observation enables us to introduce a considerable simplification in the computational procedure. Let us assume for simplicity that $\left|x_{j-1}-x_{j}\right|=|b-a| / p=$ constant for $j=1(1) p$; otherwise a scaling factor $E_{j}$ will be required. Let us single out one of the subintervals defined by the partition $\Pi$, say the first one $\left[x_{0}, x_{1}\right]$. On account of Theorem 2, all components $y_{n j}(x)$ of $\mathbf{y}_{n}(x)$ can be determined as shifts of a Tau master element (see [26]) $y_{n 1}(x)$ if we only replace $x_{j-1}$ by $x_{1}$ in the left-hand side of (11-b), and $x_{j-1}$ by $x_{0}$ in its right-hand side. Then, the only basis required will be $\mathbf{v}$, defined for $x_{0} \leqslant x \leqslant x_{1}$, and the same sequence of 
canonical polynomials (see (5)) will be sufficient to construct all components of the piecewise Tau approximant $\mathbf{y}_{n}(x)$. The procedure for the construction of $y_{n 1}(x)$ could be regarded as an integration formula based on the Tau method of [25] and specifically designed for each differential equation (1)-(2). The graphs of successive segmented Tau approximants $y_{n j}(x), j=1(1) p$, will all be in the interval $\left[x_{0}, x_{1}\right]$. The ordinate at the endpoint of the graph of $y_{n j}(x)$ will be the same as that of $y_{n j+1}\left(x_{0}\right)$. Sliding these graphs in the direction of $\overline{x_{0} x_{p}}$ we obtain the graph of $\mathbf{y}_{n}(x)$.

If Remark 3 is taken into account the structure of $Z_{n}$ is greatly simplified: except for the subblock representing the boundary conditions, all blocks $\alpha_{j}^{(n)}$ are similar.

As early as 1956 Lanczos observed a significant increase in accuracy at the endpoint of the approximation range of a Tau approximant when Chebyshev polynomials are replaced by Legendre polynomials (see Lanczos [11], [12] and Luke [13]). Ortiz [26] used this property in the design of a segmented formulation of the Tau method for initial value problems, with increased accuracy at matching points. Examples given in Section 7 of this paper show that the accuracy of segmented Tau approximants for linear and nonlinear boundary value problems improves by the use of the technique of increased accuracy at matching points. Theoretical results in this direction have been recently reported by Freilich and Ortiz [7] and by Namasivayam and Ortiz [16]. As collocation is a special realization of the Tau method, where the collocation nodes are the zeros of $H_{n}(x)$, it is clear that Lanczos' observation, the results reported in this paper, and those of [24] and [26] apply to the collocation method.

6. Nonlinear Boundary Value Problems and the Tau Method. The numerical solution of nonlinear boundary value problems with the recursive formulation of the Tau method [24] is based on the approximation of the solution of the nonlinear problem by a sequence of Tau approximants $y_{n, k}(x)$ of linear boundary value problems with variable coefficients. Each of the $y_{n, k}(x)$ is used, in an iterative cycle, to represent the nonlinear terms of the given differential equation, and gives a new $y_{n, k+1}(x)$. The fixed point of such a sequence is, under convergence conditions depending on the linearization scheme used, the function $y(x)$, solution of the initial problem. Details of this procedure can be found in Ortiz [28]. In practice such a process is only repeated a small number, $N$, of times and stopped when the maximum difference between the coefficients of two successive approximations is smaller than a given tolerance parameter $T$, specified beforehand. A test is also made on the size of the perturbation term corresponding to that approximation. If the sequence of approximants does not reach the tolerance parameter $T$ after $N$ cycles, either the degree of the Tau approximants is increased, the initial approximation redefined or the interval segmented by using the technique sketched in Section 5 . The initial approximation is usually chosen to be a polynomial satisfying (2). In some special cases the differential equation allows for the immediate determination of an algebraic curve with a contact of order $\nu-1$ with the solution $y(x)$ which is equally effective. For instance, a tangent to $y(x)$ in the case of a second order differential equation. The technique of increased convergence at matching points improves the accuracy of numerical results, as will be shown in Section 7. 
Remark 4. In the case of nonlinear boundary value problems the piecewise Tau approximants $y_{n j, k}(x)$, at stage $k$ of the process, satisfy different differential equations in each subinterval $\left[x_{j-1}, x_{j}\right], j=1(1) p$.

\section{Numerical Examples.}

(1) Dirichlet and Neumann Linear Boundary Value Problems. Let us consider the problem (see de Boor and Swartz [4]):

$$
\left\{\begin{array}{l}
D y(x):=y^{\prime \prime}(x)-4 y(x)=4 \cosh 1, \\
y(0)=y(1)=0, \quad 0 \leqslant x \leqslant 1
\end{array}\right.
$$

for which results obtained by using finite difference residual correction of a collocation solution constructed with four cubic splines centered at the points 0 , $1 / 3,2 / 3$, and 1 , have been reported recently by Oliveira [18]. In Table 1 we are only concerned with global Tau approximants defined for $0 \leqslant x \leqslant 1$. For both the differential form (14) and the corresponding integrated form we compare the first Tau correction, defined by $\left[e_{n}(x)\right]_{n+1}$ for $n=3(2) 9$, with the exact error. We wish to remark that the integrated form is never constructed, its Tau approximant is computed by using the result of (8).

TABle 1

Global Tau approximants and their corrections

\begin{tabular}{|c|c|c|c|c|}
\hline \multirow{2}{*}{ Degree } & \multicolumn{2}{|c|}{ DIFFERENTIAL FORM } & \multicolumn{2}{c|}{ INTEGRATED FORM } \\
\cline { 2 - 5 } & First Tau Correction & Exact Error & First Tau Correction & Exact Error \\
\hline 3 & $7.369 \times 10^{-2}$ & $7.415 \times 10^{-2}$ & $9.866 \times 10^{-3}$ & $9.940 \times 10^{-3}$ \\
5 & $4.576 \times 10^{-4}$ & $4.589 \times 10^{-4}$ & $7.358 \times 10^{-5}$ & $7.390 \times 10^{-5}$ \\
7 & $1.275 \times 10^{-6}$ & $1.278 \times 10^{-6}$ & $3.172 \times 10^{-7}$ & $3.181 \times 10^{-7}$ \\
9 & $3.378 \times 10^{-9}$ & $3.384 \times 10^{-9}$ & $9.048 \times 10^{-10}$ & $9.064 \times 10^{-10}$ \\
\hline
\end{tabular}

In Table 2 we present the same information when segmentation is used: four Tau approximants are constructed over subintervals of $[0,1]$ of equal length. We remark that for $n \geqslant 4$ the approximation of the differential form is more accurate than that of the integrated form.

TABLE 2

Four piecewise Tau approximants over the equally

segmented interval $0 \leqslant x \leqslant 1$ and their corrections

\begin{tabular}{|c|c|c|c|c|}
\hline \multirow{2}{*}{ Degree } & \multicolumn{2}{|c|}{ DIFFERENTIAL FORM } & \multicolumn{2}{c|}{ INTEGRATED FORM } \\
\cline { 2 - 5 } & First Tau Correction & Exact Error & First Tau Correction & Exact Error \\
\hline 3 & $2.072 \times 10^{-3}$ & $2.073 \times 10^{-3}$ & $2.016 \times 10^{-3}$ & $2.032 \times 10^{-3}$ \\
4 & $1.141 \times 10^{-5}$ & $1.196 \times 10^{-5}$ & $1.405 \times 10^{-5}$ & $1.644 \times 10^{-5}$ \\
5 & $5.448 \times 10^{-7}$ & $5.470 \times 10^{-7}$ & $2.373 \times 10^{-6}$ & $2.386 \times 10^{-6}$ \\
6 & $2.115 \times 10^{-9}$ & $2.235 \times 10^{-9}$ & $1.163 \times 10^{-8}$ & $1.282 \times 10^{-8}$ \\
7 & $1.192 \times 10^{-10}$ & $1.196 \times 10^{-10}$ & $1.181 \times 10^{-9}$ & $1.186 \times 10^{-9}$ \\
8 & $3.766 \times 10^{-13}$ & $3.908 \times 10^{-13}$ & $4.440 \times 10^{-12}$ & $4.750 \times 10^{-12}$ \\
\hline
\end{tabular}


In Table 3 we attempt a ranking of our global and segmented approximations before and after correction with the Tau estimator $\left[e_{n}(x)\right]_{n+1}$. After correction, a global Tau approximant of the solution of (14) is more accurate than the piecewise polynomial approximation obtained by Oliveira [18] by using collocation with four cubic splines and a finite difference correction.

If four cubic Tau approximants are corrected with the Tau estimator, the accuracy over Oliveira's approximation increases by a factor of $3.169 \times 10^{-3}$. By using the technique of increased accuracy at matching points, which accounts for a switch from Chebyshev to Legendre polynomials in the basis $\mathbf{v}$, that factor, now $1.619 \times$ $10^{-4}$, would show an even higher accuracy.

TABLE 3

$A$ hierarchy of global and segmented approximants

of the boundary value problem (14)

\begin{tabular}{|l|c|c|}
\hline \multicolumn{1}{|c|}{ Numerical technique } & Maximum Abs. Error & Type of Approximant \\
\hline $\begin{array}{l}\text { Corrected piecewise cubic differen- } \\
\text { tial Tau with the use of the tech- } \\
\text { nique of increased accuracy at } \\
\text { matching points }\end{array}$ & $5.8 \times 10^{-7}$ & Segmented \\
$\begin{array}{l}\text { Corrected piecewise cubic differen- } \\
\text { tial Tau }\end{array}$ & $1.1 \times 10^{-5}$ & Segmented \\
$\begin{array}{l}\text { Corrected piecewise cubic integra- } \\
\text { ted Tau }\end{array}$ & $1.4 \times 10^{-5}$ & Segmented \\
$\begin{array}{l}\text { Corrected cubic global integrated } \\
\text { Tau }\end{array}$ & $7.4 \times 10^{-5}$ & Non-segmented \\
$\begin{array}{l}\text { Piecewise cubic differential Tau } \\
\text { with the use of the technique of } \\
\text { increased accuracy at matching } \\
\text { points }\end{array}$ & $1.6 \times 10^{-4}$ & Segmented \\
$\begin{array}{l}\text { Corrected cubic global differential } \\
\text { Tau }\end{array}$ & $4.6 \times 10^{-4}$ & Non-segmented \\
$\begin{array}{l}\text { Finite differences corrected collo- } \\
\text { cation with four cubic splines [18] }\end{array}$ & $3.6 \times 10^{-3}$ & Segmented \\
$\begin{array}{l}\text { Piecewise cubic differential Tau } \\
\text { Cubic global integrated Tau }\end{array}$ & $4.0 \times 10^{-3}$ & Segmented \\
$\begin{array}{l}\text { Collocation with cubic splines [18] } \\
\text { Cubic global differential Tau }\end{array}$ & $1.0 \times 10^{-2}$ & Non-segmented \\
\end{tabular}

Remark 4. The accuracy obtained with the Tau approximant at the top of Table 3 $\left(5.827 \times 10^{-7}\right)$ is almost identical to the upper bound for the best uniform segmented approximation of the exact solution $y(x)$ by means of four algebraic polynomials of degree four, which is the degree of the corrected Tau pieces. The upper bound was estimated on the basis of Lagrange's error formula (see Meinardus [15]). Therefore, it seems possible to use segmented Tau approximants as an initial guess for the construction of segmented best approximations. 
A similar linear boundary value problem, now with Neumann conditions, and over a longer interval is:

$$
\left\{\begin{array}{l}
D y(x):=y^{\prime \prime}(x)-y(x)=1 \\
y(0)=0, \quad y^{\prime}(20)=1, \quad 0 \leqslant x \leqslant 20
\end{array}\right.
$$

considered in detail by Scott [35] with finite difference techniques.

Table 4 displays the maximum of the value of the first Tau estimator and of the exact absolute error for some of the Tau approximants considered before. They have been constructed either over [0,20], or over four equal and consecutive subintervals of $[0,20]$. In this, as in many other Neumann problems, the differential form gives consistently better results than the integrated one, even for small values of $n$ (see Onumanyi [20]).

TABLE 4

Tau approximation of a Neumann problem over a large interval

\begin{tabular}{|c|c|c|c|c|}
\hline \multirow{2}{*}{ Approximant } & \multicolumn{2}{|c|}{$n=7$} & \multicolumn{2}{c|}{$n=8$} \\
\cline { 2 - 5 } & Exact error & Tau estimator & Exact error & Tau estimator \\
\hline Global integral Tau & $2.1 \times 10^{-1}$ & $2.1 \times 10^{-1}$ & $5.4 \times 10^{-2}$ & $5.3 \times 10^{-2}$ \\
Global differential Tau & $1.1 \times 10^{-1}$ & $1.3 \times 10^{-1}$ & $2.1 \times 10^{-2}$ & $2.3 \times 10^{-2}$ \\
Piecewise integral Tau & $8.8 \times 10^{-4}$ & $8.7 \times 10^{-4}$ & $1.5 \times 10^{-4}$ & $1.4 \times 10^{-4}$ \\
Piecewise differential & $1.4 \times 10^{-4}$ & $1.3 \times 10^{-4}$ & $2.4 \times 10^{-5}$ & $2.4 \times 10^{-5}$ \\
Tau & & & & \\
\hline
\end{tabular}

(2) A Stiff Boundary Value Problem With Nonpolynomial Coefficients. If coefficients or the right-hand side of a differential equation are not polynomials, the Tau method can be used to find polynomial approximations of them. The same applies to transcendental nonlinear terms (see for example Ortiz and Samara [33]).

Let us consider the singular perturbation boundary value problem

$$
\left\{\begin{array}{l}
D y(x):=y^{\prime \prime}(x)-P y(x)=\cos x, \\
y(0)=y(\pi / 2)=1, \quad 0 \leqslant x \leqslant \pi / 2
\end{array}\right.
$$

(see Guderley [8]). The nonpolynomial term will be replaced by a polynomial approximation of degree 14 which, in this case, we generate with the present Tau method technique.

The graph of the solution $y(x)$ of the singularly perturbed boundary value problem (15) is, for large $P$, close to $y=0$ inside the interval $(0, \pi / 2)$, and jumps to $y=1$ when $x$ approaches either $x=0$ or $x=\pi / 2$ (see Figure 1).

We have computed Tau approximations of $y(x)$ for $P=1000$ and for $P=10000$. They are global Tau approximations of a moderately large degree $(n=27)$ and segmented Tau approximants of a lower degree $(n=14)$. The ability of these approximations to follow the rapid variation of $y(x)$ near the endpoints of the interval $[0, \pi / 2]$ is reported in Table 5 for both Chebyshev and Legendre perturbation terms. The graphs of the two segmented Tau approximants for $P=1000$ and $P=10000$ are reproduced in Figure 1 . 


\section{TABLE 5}

Tau approximation of a stiff boundary value problem

with a nonpolynomial term (15)

\begin{tabular}{|c|c|l|c||c|}
\hline Stiffness & $\begin{array}{c}\text { Degree of } \\
\text { approximant }\end{array}$ & $\begin{array}{l}\text { Perturbation } \\
\text { term used }\end{array}$ & $\begin{array}{l}\text { Nr. of equal } \\
\text { subintervals }\end{array}$ & Max. Abs. Error \\
\hline $10^{3}$ & 27 & Chebyshev & 1, no segm. & $1.0 \times 10^{-7}$ \\
$10^{3}$ & 14 & Chebyshev & 8 & $2.0 \times 10^{-10}$ \\
$10^{3}$ & 14 & Legendre & 8 & $1.1 \times 10^{-10}$ \\
\hline $10^{4}$ & 28 & Chebyshev & 1, no segm. & $1.0 \times 10^{-2}$ \\
$10^{4}$ & 14 & Chebyshev & 12 & $7.8 \times 10^{-7}$ \\
$10^{4}$ & 14 & Legendre & 12 & $4.4 \times 10^{-7}$ \\
\hline
\end{tabular}

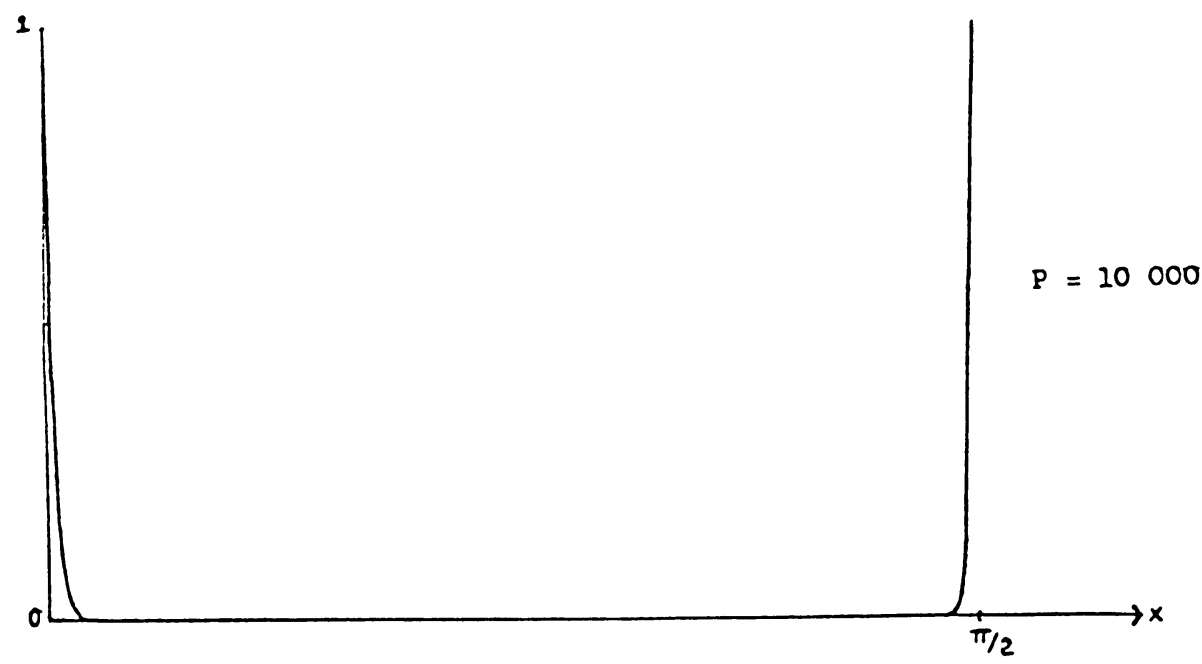

FIGURE 1

Graphs of the exact solution of the singularly perturbed boundary value problem (15), for $P=10000$, and of a segmented Tau approximant of degree 14, in the construction of which the technique of increased accuracy at matching points has been used. The maximum deviation between the two curves, seen as one in the graph, is $4.4 \times 10^{-7}$ (see Table 5).

(3) Control of the Segmentation Process With $\left[e_{n}(x)\right]_{m}$ in an Adaptive Formulation of the Tau Method. We now consider the singular perturbation boundary value problem with a boundary layer at $x=1$ defined by the differential equation

$$
\left\{\begin{array}{l}
D y(x):=-y^{\prime \prime}(x)+\frac{d}{d x}[P(1-c x) y(x)]=0, \\
y(0)=1, \quad y^{\prime}(1)=0, \quad 0 \leqslant x \leqslant 1,
\end{array}\right.
$$

where $c=0.98$ and $P=1000$. The graph of the solution is a spike, symmetric about $x=1$, extending in the $y$-axis direction from 1 up to about 50 when $x$ is in $[0,2]$. Problem (16) has been discussed numerically in a recent paper by Barrett and Morton [1], with a quasi-symmetrization technique based on Galerkin's method with a piecewise linear trial space; further references can be found in that paper.

We have constructed for this problem global Tau approximants of degrees 27 and 28 , then a segmented piecewise Tau approximant of degree 7 defined over four equal 
subintervals of $[0,1]$. The Tau estimator $\left[e_{n}(x)\right]_{m}$, with $m=n+1$, was used to detect the region of rapid variation of the last segmented approximant, and then a new nonuniform segmentation was introduced, with nodes in $x=0.0 ; 0.7 ; 0.8 ; 0.9$ and 1.0. A segmented Tau piecewise approximant, of degree 7 over each subinterval, was constructed. In Table 6 we reproduce our numerical results; we have also included the values of the exact solution up to $2 \mathrm{D}$. Agreement to $2 \mathrm{D}$ is provided by the Tau approximant.

\section{TABLE 6}

Use of the Tau estimator $\left[e_{n}(x)\right]_{m}$ for the generation of $a$ nonuniform segmentation strategy, better adapted to the singular nature of the solution of the boundary layer problem (16); $m=n+1$.

\begin{tabular}{|l|c|c|c|c|c|}
\hline Type of approximant & Nr. of pieces & $x=0.7$ & $x=0.8$ & $x=0.9$ & $x=1.0$ \\
\hline Global Tau, $n=27$ & 1 & 3.10 & 4.73 & 9.32 & 49.99 \\
Global Tau, $n=28$ & 1 & 3.21 & 4.73 & 9.32 & 49.94 \\
$\begin{array}{l}\text { Segmented piecewise Tau, } \\
\text { uniform segmentation, } n=7\end{array}$ & 4 & 3.22 & 4.48 & 8.40 & 48.21 \\
$\begin{array}{l}\text { Segmented piecewise Tau, } \\
\text { adapted-nonuniform segmen- } \\
\begin{array}{l}\text { tation, controlled by the } \\
\text { Tau estimator }\left[e_{n}(x)\right]_{m}, n=7\end{array}\end{array}$ & 4 & 3.21 & 4.73 & 9.31 & 49.95 \\
\hline EXACT RESULTS & & 3.21 & 4.73 & 9.31 & 49.95 \\
\hline
\end{tabular}

Results reported in [1] with quasi-symmetrization lead to large errors: +0.14 and -0.52 , near $x=1 \quad(x=0.8$ and 0.9 respectively). A comparison between the upwinding technique of [2] and Tau method approximants is also favorable to the recursive formulation of the Tau method (see Ortiz [29]).

(4) Tau Approximate Solution and Error Estimation of a Nonlinear Boundary Value Problem With Global and Segmented Approximants. The numerical solution of nonlinear differential equations with the recursive formulation of the Tau method is reduced to the approximate solution of a sequence of linear problems, as indicated in Section 6, that is, to problems of the type considered before. However, we shall discuss explicitly a nonlinear problem with a singularity of the first kind proposed by Russell and Shampine [34], for which results with the finite difference techniques of de Hoog and Weiss [5] are available,

$$
\begin{aligned}
\frac{d}{d x}\left[\begin{array}{c}
y_{1}(x) \\
-y_{2}(x)
\end{array}\right] & =\frac{1}{x}\left[\begin{array}{ll}
0 & 0 \\
0 & 2
\end{array}\right]\left[\begin{array}{l}
y_{1}(x) \\
y_{2}(x)
\end{array}\right]+\left[\begin{array}{l}
y_{2}(x) \\
y_{1}^{5}(x)
\end{array}\right] \\
{\left[\begin{array}{l}
y_{1}(1) \\
y_{2}(0)
\end{array}\right] } & =\left[\begin{array}{c}
\sqrt{.75} \\
0
\end{array}\right], \quad 0 \leqslant x \leqslant 1
\end{aligned}
$$

which we treat as the second order boundary value problem

$$
\left\{\begin{array}{l}
D y(x):=y^{\prime \prime}(x)+(2 / x) y^{\prime}(x)+y^{5}(x)=0 \\
y^{\prime}(0)=0, \quad y(1)=\sqrt{0.75}, \quad 0 \leqslant x \leqslant 1
\end{array}\right.
$$


A direct approximation of systems of differential equations with the recursive formulation of the Tau method is also possible if vector canonical polynomials are used; see [6] for details on this approach. The exact solution of (17)-(18) is known to be $y(x)=\left(1+x^{2} / 3\right)^{-1 / 2}$, which makes it possible to compare the error of our Tau approximations of $y(x)$ with the estimation of that error provided by the Tau estimator $\left[e_{n}(x)\right]_{m}$.

We fix the tolerance parameter $T$ (see Section 3 ) equal to $10^{-6}$ and the maximum permissible number of cycles $N$ equal to 5 which turns out to be sufficiently large. Table 7 displays the errors of approximation of a global and a segmented Tau approximant, the latter over the subintervals $[0,0.5]$ and $[0.5,1]$.

\section{TABLE 7}

Maximum absolute error of approximation for the nonlinear boundary value problem (18) when approximated with global and segmented Tau approximants.

\begin{tabular}{|c|c|c|c|c|}
\hline \multirow{2}{*}{$\begin{array}{c}\text { Degree of the } \\
\text { Tau approximant }\end{array}$} & \multicolumn{2}{|c|}{ Global Approximation } & \multicolumn{2}{c|}{ Segmented approximation } \\
\cline { 2 - 5 } & Max. Abs. Error & $\begin{array}{c}\text { Nr. of cycles } \\
\text { required }\end{array}$ & Max. Abs. Error & $\begin{array}{c}\text { Nr. of cycles } \\
\text { required }\end{array}$ \\
\hline 4 & $8.7 \times 10^{-4}$ & 3 & $7.8 \times 10^{-5}$ & 3.3 \\
5 & $6.0 \times 10^{-5}$ & 3 & $1.6 \times 10^{-6}$ & 3.3 \\
6 & $6.6 \times 10^{-6}$ & 3 & $1.0 \times 10^{-6}$ & 3.3 \\
\hline
\end{tabular}

The Tau error estimator $\left[e_{n}(x)\right]_{m}$, applied to both global and segmented approximants, leads to remarkably accurate estimations of the exact error. Table 8 reproduces results for $n=4, m=n+1$ and $n+2$, with global and segmented Tau approximants. For $m=n+1$ the order of the error is correctly estimated in both cases.

\section{TABLE 8}

Error estimation of global and segmented Tau approximants of the nonlinear singular boundary value problem (18), given by the Tau estimator $\left[e_{n}(x)\right]_{m}$ for $n=4$.

\begin{tabular}{|c|c|c|c|}
\hline \multicolumn{2}{|c|}{ Global Tau approximant } & \multicolumn{2}{c|}{ Segmented Tau approximants } \\
\hline$m$ & $\begin{array}{l}\text { Difference between the } \\
\text { exact and estimated errors }\end{array}$ & $m, m$ & $\begin{array}{c}\text { Difference between the } \\
\text { exact and estimated errors }\end{array}$ \\
\hline$n+1$ & $6.01 \times 10^{-5}$ & $n+1, n+1$ & $1.57 \times 10^{-6}$ \\
$n+2$ & $6.50 \times 10^{-6}$ & $n+2, n+2$ & $1.05 \times 10^{-6}$ \\
\hline
\end{tabular}

The nonlinear system of differential equations

$$
\frac{d}{d x}\left[\begin{array}{l}
y_{1}(x) \\
y_{2}(x)
\end{array}\right]=\left[\begin{array}{c}
y_{2}(x) \\
(1 / \varepsilon)\left[y_{1}^{2}(x)-x^{2}\right] y_{2}(x)
\end{array}\right], \quad\left[\begin{array}{c}
y_{1}(-1) \\
y_{1}(0)
\end{array}\right]=\left[\begin{array}{c}
0.96 \\
0.001
\end{array}\right],
$$

$-1 \leqslant x \leqslant 0$, is a nonlinear model of a singularly perturbed differential equation where the leading coefficient of the reduced equation has turning points, the location of which depends on the unknown function. It is a stiff problem with a multiplicity 
of solutions which has been treated by Kedem [9] by collocation $(\varepsilon=15)$, and by Ortiz [29] with the Tau method (global approximant); further references can be found in these papers. High-order boundary value problems for differential equations are considered in [21] (global approximant). Further numerical results on a large variety of problems where the technique discussed in this paper has been successfully applied are reported in Onumanyi [19]-[20].

Mathematics Department

Imperial College

London, England

1. J. W. Barrett \& K. W. Morton, Optimal Finite Element Solutions to Diffusion-Convection Problems in One Dimension, University of Reading, Numerical Analysis Report 3/78, 1978.

2. I. Christie, D. F. Griffith, A. R. Mitchell \& O. C. Zienkiewicz, "Finite element methods for second order differential equations with significant derivatives," Internat. J. Numer. Methods Engrg., v. 10, 1976, pp. 1389-1396.

3. M. R. Crisci \& E. Russo, "An extension of Ortiz' recursive formulation of the Tau method to certain linear systems of ordinary differential equations," Math. Comp., v. 41, 1983, pp. 27-42.

4. C. DE BOoR \& B. SWARTz, "Collocation at Gaussian points," SIAM J. Numer. Anal., v. 10, 1973, pp. 582-606.

5. F. R. DE Hoog \& R. WEISs, "Difference methods for boundary value problems with a singularity of the first kind," SIAM. J. Numer. Anal., v. 13, 1976, pp. 775-813.

6. J. H. Freilich \& E. L. ORTIZ, "Numerical solution of systems of ordinary differential equations with the Tau method: An error analysis," Math. Comp., v. 39, 1982, pp. 467-479.

7. J. H. FreILICH \& E. L. ORTIZ, On the Error Analysis of a Vector Rational Approximation of the Solution of a System of Ordinary Differential Equations With the Tau Method, Imperial College Research Report NAS 75, 1975, pp. 1-14.

8. K. G. GUDERLEY, "A unified view of some methods for stiff two-point boundary value problems," SIAM Rev., v. 17, 1975, pp. 416-442.

9. G. KEDEM, "A posteriori error bounds for two-point boundary value problems," SIAM J. Numer. Anal., v. 18, 1981, pp. 431-448.

10. C. LANCZos, "Trigonometric interpolation of empirical and analytical functions," J. Math. Phys., v. 17, 1938, pp. 123-199.

11. C. Lanczos, Applied Analysis, Prentice-Hall, Englewood Cliffs, N. J., 1956.

12. C. Lanczos, "Legendre versus Chebyshev polynomials," Topics in Numerical Analysis (J. J. H. Miller, Ed.), Academic Press, New York, 1973.

13. Y. L. LUKE, The Special Functions and Their Approximations, Vols. I and II. Academic Press, New York, 1969.

14. P. Llorente \& E. L. Ortiz, "Sur quelques aspects algébriques d'une méthode de M. Lanczos," Math. Notae, v. 21, 1968, pp. 17-21.

15. G. MeInARDus, Approximation of Functions: Theory and Numerical Methods, Springer-Verlag, Berlin, 1967.

16. S. Namasivayam \& E. L. OrTiz, Perturbation Terms and Approximation Error in the Numerical Solution of Differential Equations With the Tau Method, Imperial College, Research Report NAS 05-09-81, 1981, pp. 1-5.

17. S. Namasivayam \& E. L. ORTIZ, Approximate coefficients and approximation error in the numerical solution of differential equations, with an application to the Tau method, Research Report NAS 04-08-82.

18. F. A. OliveIRA, "Collocation and residual correction," Numer. Math., v. 36, 1980, pp. 27-31.

19. P. ONUMANY, Numerical Experiments With Some Nonlinear Ordinary Differential Equations Using the Tau Method, M. Sc. Thesis, Imperial College, 1978.

20. P. Onumany, Numerical Solution of Nonlinear Boundary Value Problems With the Tau Method, Ph.D. Thesis, Imperial College, 1981.

21. P. ONUMANYI \& E. L. ORTIZ, "Numerical solution of high order boundary value problems for ordinary differential equations with an estimation of the error," Internat. J. Numer. Methods Engrg., v. 18, 1982, pp. 775-781.

22. P. Onumany, E. L. Ortiz \& H. Samara, "Software for a method of finite approximations for the numerical solution of differential equations," Appl. Math. Modelling, v. 5, 1981, pp. 282-286.

23. E. L. ORTIZ, On the Generation of the Canonical Polynomials Associated With Certain Linear Differential Operators, Imperial College Research Report NAS 64, 1964, pp. 1-22. 
24. E. L. Ortiz, “The Tau method,” SIAM J. Numer. Anal., v. 6, 1969, pp. 480-492.

25. E. L. ORTIz, "Canonical polynomials in the Lanczos Tau method," Studies in Numerical Analysis (B. Scaife, Ed.), Academic Press, New York, 1974, pp. 73-93.

26. E. L. ORTIZ, "Step by step Tau method, Piecewise polynomial approximations," Comput. Math. Appl., v. 1, 1975, pp. 381-392.

27. E. L. OrTIz, Sur Quelques Nouvelles Applications de la Méthode Tau, Séminaire Lions, Analyse et Contrôle de Systèmes, IRIA, Paris, 1975, pp. 247-257.

28. E. L. ORTIZ, "On the numerical solution of nonlinear and functional differential equations with the Tau method," in Numerical Treatment of Differential Equations in Applications (R. Ansorge and W. Törnig, Eds.), Lecture Notes in Math., Vol. 679, Springer-Verlag, Berlin, 1978, pp. 127-139.

29. E. L. ORTIz, "Polynomial and rational approximation of boundary layer problems with the Tau method," in Boundary and Interior Layers-Computational and Asymptotic Methods (J. J. H. Miller, Ed.), Boole Press, Dublin, 1980, pp. 387-391.

30. E. L. Ortiz \& A. Pham Ngoc Dinh, “On the convergence of the Tau method for nonlinear differential equations of Riccati’s type," Nonlinear Analysis, 1984. (In press.)

31. E. L. Ortiz \& A. Pham Ngoc Dinh, "An error analysis of the Tau method for a class of singularly perturbed problems for differential equations," Math. Methods Appl. Sci., 1984. (In press.)

32. E. L. Ortiz, F. J. Rodriguez Cañizares \& W. F. C. PUrser, Automation of the Tau Method, Imperial College Research Report NAS 01-72, 1972, pp. 1-54. (Presented to the Conference on Numerical Analysis organized by the Royal Irish Academy, Dublin, 1972.)

33. E. L. Ortiz \& H. SAmara, "An operational approach to the Tau method for the numerical solution of nonlinear differential equations," Computing, v. 27, 1981, pp. 15-25.

34. R. D. Russell \& L. F. Shampine, “A collocation method for boundary-value problems," Numer. Math., v. 19, 1972, pp. 1-28.

35. M. R. ScoTt, "On the conversion of boundary-value problems into stable initial-value problems via several invariant imbedding algorithms," in Numerical Solution of Boundary Value Problems for Ordinary Differential Equations (A. K. Aziz, Ed.), Academic Press, New York, 1975, pp. 89-146.

36. H. J. STETTER, "Economical global error estimation," in Stiff Differential Equations (R. A. Willoughby, Ed.), Plenum Press, New York, 1974, pp. 245-258.

37. P. ZADUNAISKY, "On the estimation of errors in the numerical integration of ordinary differential equations,” Numer. Math., v. 27, 1976, pp. 21-39. 